\title{
Über Nicht-Krankheiten und Medikalisierung
}

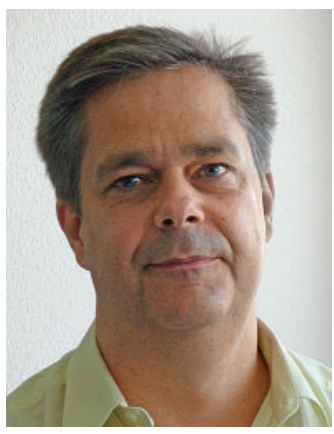

Eberhard Wolff
Kommt Ihnen spontan eine Krankheit in den Sinn, die «eigentlich» gar keine ist? Das British Medical Journal veröffentlichte 2002 das Ergebnis einer entsprechenden Umfrage [1]*. "Alter» hiess der Gewinner. Die meisten der geschätzten Leserinnen und Leser werden zustimmen, dass Alter eigentlich keine Krankheit ist, und sie werden trotzdem nicht gänzlich darauf verzichten wollen, altersbezogene Symptome zu behandeln.

Mit ihrer «Nicht-Krankheiten»-Hitparade wollte das BMJ zum einen zeigen, wie «slippery» der Krankheitsbegriff ist. Zum anderen wollte es auf die Tendenz aufmerksam machen, dass menschliche Probleme zunehmend als Krankheiten klassifiziert werden. Ein anderes Beispiel wäre das Phänomen der «Sucht», das sich, vereinfacht, von einer Sünde zu einer Krankheit entwickelt hat. Aktueller Medienstar hierfür: «Sexsucht» [2].

Dahinter steht der allgemeine Trend, dass immer mehr gesellschaftliche Phänomene «medikalisiert», d. h. medizinisch interpretiert und angegangen sowie unter den Einflussbereich des Medizinsystems gestellt werden: ein gefundenes Thema für die jüngste SAMW/NEK-Tagung [3]. Deren Eröffnungsbeispiel: Eisenmangel. Der ist heute eine Trend-Diagnose. Müdigkeitssymptome werden zum Teil vorschnell mit Eisenmangel erklärt, können aber vielfältige andere, medizinische wie nichtmedizinische Ursachen haben. Mehr noch: Die teurere intravenöse Therapie ist «auf dem Weg, sich zu einer teuren Lifestyle-Droge zu entwickeln» [4].

Ein ökonomischer Blick sieht Medikalisierung im steigenden Anteil der Medizinausgaben am Bruttoinlandsprodukt abgebildet. Genauer: als Verschiebung der Konsumnachfrage in Richtung Gesundheit, u.a. weil die Nachfrage nach anderen Konsumgütern gesättigt sei [5].

Der Vormarsch der Medizin seit der Aufklärung mit ihrem Zugriff auf den Alltag hatte und hat aber immer auch soziokulturelle Hintergründe. Die Onaniehysterie war ebenso von gesellschaftlichen Wertvorstellungen geprägt wie die eugenischen Degenerationsängste - und das heutige Sprechen über das Übergewicht ist es auch [6]. Jugendlichkeit, Leistungsfähigkeit oder Schönheit sind heute dominante gesellschaftliche Leitideen, für deren Erreichung die Medizin ihr gesamtes Instrumentarium zur Verfügung stellt, nicht nur Neuroenhancer und Botox. Zudem wird der Medizin als Expertensystem eine Autorität zuerkannt, wie sie in der Vormoderne die
Religion innehatte. Gerade hier zeigen sich aber auch Grenzen der Medikalisierung: Die Autorität professioneller medizinischer Eliten (vulgo: der Ärzte) hat ihren Zenit seit einigen Jahrzehnten überschritten. Und die Entpathologisierung der Homosexualität ist zumindest ein Einzelbeispiel aktiver Demedikalisierung gegen den Mainstream, der in Richtung «Gesundheitsgesellschaft» führt [7].

Es griffe allerdings zu kurz, den komplexen Phänomenen neu aufkommender Krankheiten mit dem Schlagwort «Mode-Krankheit» einfach die Existenzberechtigung abzusprechen. «Burn-out» hat sich als neues Krankheitskonzept in den letzten Jahrzehnten durchaus stabilisiert, obwohl es nicht wie beim «ADHS» durch eine gängige Therapie gestützt wird. Ein Grund mag sein, dass sich Betroffene darin verstanden fühlen und es als Metapher für Zeitkritik, als Protesthaltung eingesetzt werden kann. Werden Menschen mittels «Burn-out» also medikalisiert? Wird die Gesellschaft damit medikalisiert? Wird damit umgekehrt die Medizin vergesellschaftlicht? Oder ist das Gesellschaftsphänomen «Burn-out» eine Chance, sich aus stigmatisierenden KrankheitsSchablonen wie «Depression» zu befreien [8]?

Sicher, «die» Medizin hat ihren Einfluss ausgeweitet. Und so lag die politische Frage hinter der Tagung auf der Hand: Wo sollen/müssen der Medikalisierung Grenzen gesetzt werden? Ethiker und Politiker hätten gerne Werkzeuge, mit denen sie einen eindeutigen Gartenzaun zwischen «unangemessener Medikalisierung» [9] und einer «eigentlichen» Medizin ziehen können, um Leistungskataloge anzupassen oder Standesregeln $\mathrm{zu}$ definieren. Vor einigen Jahrzehnten hätte die Forschung noch eindeutigere Antworten gegeben. Da wurde die Medikalisierung bisweilen als Moloch zunehmender Ärztemacht dämonisiert, der die Gesellschaft erobert. «Gut» und «böse» waren klarer verteilt. Heute wird Medikalisierung eher als Konsequenz unserer Gesellschaft gelesen, als selbstverständlicher, auch gewollter Alltag. Die Beispiele der Tagung wie das «Burn-Out» (oder das «Enhancement») zeigen in dieser Perspektive eher, dass es keine «eigentliche» Medizin ohne gesellschaftliche Einflüsse gibt und die Übergänge viel zu fliessend und die Bereiche zu verflochten sind, um klare Grenzen zu ziehen. So wie «Alter» keine Krankheit ist - und irgendwo doch eine: «slippery» eben. Keine einfache Sache in der Praxis.

Eberhard Wolff** 


\section{Referenzen}

1 Smith R. In search of «non-disease». BMJ 2002;324: 883-5.

2 http://blog.tagesanzeiger.ch/mamablog/index.php/ 17761/mythos-sexsucht/

3 Medizin total. Die Medikalisierung des Alltags als ethisches Problem. Organisiert von der Akademie der Medizinischen Wissenschaften (www.samw.ch) und der Nationalen Ethikkommission (www.nek-cne.ch). Programm und Präsentationen unter www.samw.ch/ de/Projekte/Oekonomisierung-Medizin.html.

Viele Inhalte des Beitrags sind den Tagungsreferaten entlehnt. Zum Hören auch der Radiobeitrag zur Tagung von Odette Frey unter www.drs.ch/www/de/ drs/sendungen/echo-der-zeit/2646.sh10185046.html Zum Thema auch der aktuelle Beitrag «Krank zu sein bedarf es wenig» von Werner Bartens in der Süddeutschen Zeitung vom 16. Juli 2011: www. sueddeutsche.de/leben/geldmacherei-mit-patientendie-krankheitsfinder-1.1120684
4 Siehe Beitrag von Franziska Demarmels Biasiutti, Bern, auf der Tagung. Roch Ogier, Genève, dagegen betonte u.a., dass der Eisenmangel keine medizinische Neuheit sei, es aber diagnostische Mittel für und Therapien gegen ihn gebe.

5 Siehe Beitrag von Stefan Felder, Basel, auf der Tagung.

6 Siehe Beitrag von Iris Ritzmann, Zürich, auf der Tagung.

7 Siehe Beitrag von Peter C. Meyer, Winterthur, auf der Tagung.

8 Siehe Beitrag Peter Schneider, Zürich, auf der Tagung sowie die anschliessende Diskussion.

9 Siehe Beitrag von Christian Kind auf der Tagung. 